\title{
Beam and target dependencies of two-kaon femtoscopic corre- lations in SELEX
}

\author{
Grigory Nigmatkulov ${ }^{1, \star ~(f o r ~ t h e ~ S E L E X ~ c o l l a b o r a t i o n) ~}$ \\ ${ }^{1}$ National Research Nuclear University MEPhI (Moscow Engineering Physics Institute), Kashirskoe highway \\ 31, Moscow, 115409, Russia
}

\begin{abstract}
The space and time extents of the particle emitting source created in collisions of particles with nuclei can be measured using a correlation femtoscopy method. In this paper we show the preliminary measurements of $K^{ \pm} K^{ \pm}$correlation functions in $600 \mathrm{GeV} / c \Sigma^{-}, \pi^{-}$and $540 \mathrm{GeV} / c \mathrm{p}$ interactions with carbon and copper nuclei. The one-dimensional correlation functions are parameterized by a Gaussian form. The dependencies of the charged kaon emitting source radii on the different $\mathrm{h}+\mathrm{A}$ collisions are presented.
\end{abstract}

\section{Introduction}

Multiparticle production in high-energy collisions of particles and/or nuclei is a basic property of the strong interaction which is described by the Quantum Chromodynamics (QCD). The formation of hadrons from quarks and gluons (hadronization) which are confined occurs on a distance scale of $1 \mathrm{fm}$. The multiparticle production is a dynamic process, evolving space and time. Correlations of particles with small relative momenta (also known as femtoscopy or HBT intensity interferometry) [1] are used to probe space-time structure of systems created in high-energy collisions. These correlations arise from Bose-Einstein enhancement of identical-boson production at low relative momenta. In this paper we report on the results of two-kaon femtoscopic analysis performed for $600 \mathrm{GeV} / c \Sigma^{-}, \pi^{-}$and $540 \mathrm{GeV} / c$ p interactions with carbon and copper nuclei.

\section{Data sample}

The SELEX (E781) experiment is a three-stage magnetic spectrometer which was designed to study hadroproduction in the forward hemisphere $\left(x_{F}>0.1\right)$ [2]. Data were taken with $600 \mathrm{GeV} / c$ negative hadron beam $\left(\simeq 50 \% \Sigma^{-}\right.$and $\left.\simeq 50 \% \pi^{-}\right)$and with $540 \mathrm{GeV} / c$ positive hadron beam $(\simeq 92 \% \mathrm{p}$ and $\left.\simeq 8 \% \pi^{+}\right)$. The beam particle was identified by a transition radiation detector and tracked by 8 beam silicon strip detectors located before the charm production target. A segmented target consists of 2 copper and 3 diamond (carbon) sheets, separated by $1.5 \mathrm{~cm}$ clearance. The total target thickness was $5 \%$ on an interaction length for protons. Produced particles were tracked in a set of 20 vertex silicon strip detectors arranged in 4 sets of planes rotated by $45^{\circ}$. The single-track efficiency of each detector

\footnotetext{
^e-mail: nigmatkulov@gmail.com,ganigmatkulov@mephi.ru
} 
was exceeding 98\%. All strip detectors have a resolution of $6.5 \mu \mathrm{m}$ ( $\mathrm{pitch} / \sqrt{12})$. The average longitudinal vertex position resolution was $270 \mu \mathrm{m}$ for primary vertex and $560 \mu \mathrm{m}$ for secondary vertex. The particle momentum was measured by deflection of the track position by two magnets in a system of proportional wire chambers (PWC). In order to achieve high-resolution momentum measurements (up to full beam energy), the beam region of PWCs was covered with double-sided silicon detectors. Momentum resolution of $100 \mathrm{GeV} / c$ track was $\sigma_{p} / p \approx 0.5 \%$. Particles with momentum above $22 \mathrm{GeV} / c$ traversed to a Ring Imaging Cherenkov detector (RICH) and identified as $\pi$, K or p. The kaon identification efficiency was over $90 \%$ above the kaon threshold $(\approx 43 \mathrm{GeV} / c)$. The average number of tracks reaching the RICH was about 5 per event. The RICH detector provided $2 \sigma \mathrm{K} / \pi$ separation up to $165 \mathrm{GeV} / c$ [4]. In order to achieve high kaon identification purity, only tracks with the momentum from 45 to $160 \mathrm{GeV} / \mathrm{c}$ were selected. Charged kaons were identified with the likelihood to be a kaon at least three times exceeding any other particle hypothesis [5]. The electrons were eliminated from the analysis using the transition radiation detector which was placed before $\mathrm{RICH}$.

To measure the femtoscopic radii dependence on the nuclear mass A, the charged particles produced in the copper and carbon targets were analyzed separately. The reconstructed $z$-position of the primary vertex required to be in vicinity of a $\mathrm{Cu}$ or $\mathrm{C}$ target and not more than $0.05 \mathrm{~mm}$ from the edge of any target [3]. Primary tracks which have vertex silicon track segment matched with downstream segments in forward spectrometers used in the analysis. Each track was extrapolated to the primary vertex to determine its transverse miss distance. Only tracks with miss distance less than $20 \mu \mathrm{m}$ were used. After applying the cuts $1065655,249210,53308 \mathrm{~K}^{+} \mathrm{K}^{+}$and $3688541,337034,48298 \mathrm{~K}^{-} \mathrm{K}^{-}$ pairs were selected for $\Sigma^{-}, \pi^{-}$and $\mathrm{p}$ beams.

\section{Femtoscopy}

The main tool of the correlation femtoscopy method is the correlation function $C(\mathbf{q})$ which is defined as a ratio $C(\mathbf{q})=A(\mathbf{q}) / B(\mathbf{q})$, where $A(\mathbf{q})$ is the distribution of two-particle relative momenta $\mathbf{q}=$ $\mathbf{p}_{\mathbf{1}}-\mathbf{p}_{\mathbf{2}}$, and $B(\mathbf{q})$ is a similar distribution in all aspects except the absence of the quantum statistical correlations. A is constructed from identical particles from the same event and B is constructed using the event mixing technique where particles of interest are combined from different events. The limited statistics available (about 50k identical-kaon pairs for $\mathrm{p}$ induced collisions) allowed us to perform the analysis using only one-dimensional correlation function $C_{2}\left(q_{i n v}\right)$. The $q_{i n v}$ is a one-dimensional Lorentz scalar $q_{\text {inv }}=\sqrt{\left(\mathbf{p}_{1}-\mathbf{p}_{2}\right)^{2}-\left(E_{1}-E_{2}\right)^{2}}$. The momentum correlations of particles emitted at nuclear distances are also influenced by the effect of final-state interaction (FSI), Coulomb and strong interactions [6]. For identical kaons, the effect of strong interaction is negligible. In order to extract the emitting source radius, $R$, the correlation function can be fitted with the parameterization assuming that the emission function describes a static source and has the Gaussian profile:

$$
C_{2}\left(q_{i n v}\right)=N\left(1-\lambda+\lambda K\left(q_{i n v}\right)\left(1+\exp \left(-R^{2} q_{\text {inv }}^{2}\right)\right)\right) D\left(q_{i n v}\right)
$$

where $N$ is a normalization factor, $\lambda$ is a correlation strength and the factor $D\left(q_{\text {inv }}\right)$ is so-called baseline distribution that takes into account non-femtoscopic correlations. The factor $K\left(q_{\text {inv }}\right)$ takes into account two charged kaon Coulomb repulsion [7, 8].

For elimination of possible biases because of the construction of reference sample, one may use Monte Carlo simulation where the quantum statistical effects are absent and build so-called double ratio:

$$
C_{2}\left(q_{i n v}\right)=\frac{A\left(q_{i n v}\right) / B\left(q_{i n v}\right)}{A^{M C}\left(q_{\text {inv }}\right) / B^{M C}\left(q_{i n v}\right)},
$$

where $A^{M C}\left(q_{i n v}\right)$ and $B^{M C}\left(q_{i n v}\right)$ are the Monte Carlo relative momentum distributions from the same and mixed events, respectively. 


\section{Results and Discussions}

The positive and negative kaon femtoscopic correlations are measured using the same detector setup, cuts and fitting procedures. This allows to compare the emitting region parameters for different $\mathrm{h}+\mathrm{A}$ collisions. The analysis was performed separately for the copper and carbon targets and $600 \mathrm{GeV} / c$ $\Sigma^{-}, \pi^{-}$and $540 \mathrm{GeV} / c \mathrm{p}$ beams. In order to construct the uncorrelated reference sample the event mixing technique was used. Kaons from adjacent events were grouped by the production target and beam particle. Relative momentum distributions were combined in the numerator and denominator separately for copper and carbon targets before constructing the ratios. The correlation functions were normalized such that the $C\left(q_{i n v}\right)=1$ for the $0.6<q_{i n v}<0.8 \mathrm{GeV} / c$ region, where the influence of the non-femtoscopic correlations is small and the Bose-Einstein correlations are absent. The peak at $q_{\text {inv }}<0.4 \mathrm{GeV} / c$ is due to the quantum statistics correlations.

Particle pairs with small relative momentum are spatially close to each other and affected by the track reconstruction inefficiencies such as track splitting and track merging. Track merging effects suppress the correlation function at low $q_{i n v}$. To eliminate this effect, we require the tracks in a pair to be well separated (the average separation in the silicon strip detector region must be greater than $100 \mu \mathrm{m})$ in addition to the cuts used in the previous analysis [5]. The systematic uncertainty due to the two-particle effects was estimated by varying the cuts and does not exceed $8 \%$.

The smearing of single particle momenta was studied by embedding simulated kaon tracks with known momenta through the detector. Using detailed studies of the momentum resolution, we correct the correlation functions with an iterative technique similar to that used by NA44 and STAR collaborations $[9,10]$. Experimental correlation functions were corrected for momentum resolution using the expression:

$$
C_{2}^{\text {corr }}\left(q_{\text {inv }}\right)=\frac{C_{2}^{\text {uncorr }}\left(q_{\text {inv }}\right) C_{2}^{\text {unsmeared }}\left(q_{\text {inv }}\right)}{C_{2}^{\text {smeared }}\left(q_{\text {inv }}\right)},
$$

where $C_{2}^{\text {corr }}\left(q_{\text {inv }}\right)$ is a corrected correlation function and $C_{2}^{\text {uncorr }}\left(q_{\text {inv }}\right)$ represents a measured correlation function. The $C_{2}^{\text {unsmeared }}\left(q_{\text {inv }}\right)$ and $C_{2}^{\text {smeared }}\left(q_{\text {inv }}\right)$ are without and with taking into account the momentum resolution effect, respectively.

Figure 1 shows the correlation function before (black circles) and after (red circles) the correction on the momentum resolution. The momentum resolution correction affects the extracted source radii not more than on $1 \%$ and has negligible effect on the correlation strength.

In order to disentangle the correlation signals induced by non-femtoscopic correlations, the Monte Carlo (MC) generator PYTHIA-6.4.28 with Perugia 2011 tune is used [11, 12]. PYTHIA does not contain Bose-Einstein correlations and final-state interactions (Coulomb and strong) and thus will introduce only the correlations due to the energy-momentum conservation effects. In figure 2 the simulated correlation functions of positively and negatively charged kaons produced in the interactions of the negative pion beam with copper and carbon targets are compared to the measured ones. The source parameters were estimated in the following way. First, the simulated correlation functions were fitted with the first order polynomial $D\left(q_{\text {inv }}\right)=a+b q_{\text {inv }}$. Then the experimental correlation function is fitted by (1), taking the $D\left(q_{i n v}\right)$ from the PYTHIA fit. In order to estimate the systematic uncertainty, different function forms were used for the baseline function. The systematic uncertainty due to the baseline determination does not exceed $2 \%$.

Figure 3 shows the correlation functions (double ratios) obtained according to (2) for positive (black solid circles) and negative (blue empty circles) kaon pairs. The correlation functions were fitted using (1) and the non-femtoscopic term was equal to unity. Solid red and dashed magenta lines represent the correlation function fits of positive and negative kaons, respectively. 


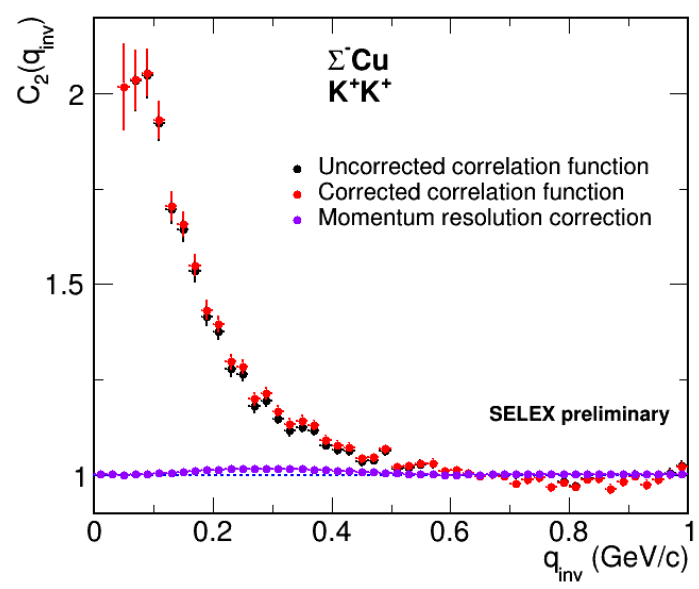

Figure 1. The positive kaon experimental correlation function measured for $\Sigma^{-} \mathrm{Cu}$ interactions before (black circles) and after (red circles) the momentum resolution correction. Magenta circles represent the correction factor due to the momentum resolution.

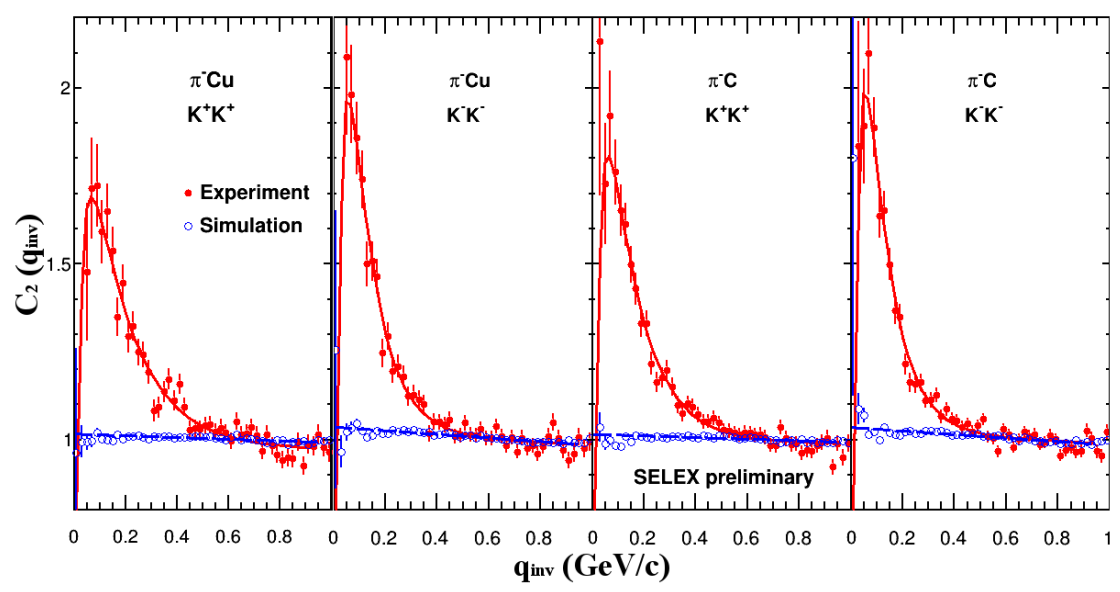

Figure 2. The experimental (red solid circles) and simulated (blue empty circles) correlation functions of positive and negative kaons obtained for $\pi^{-} C u$ and $\pi^{-} C$ interactions. The blue dashed lines represent baseline fits. The red lines are the correlation function fits using (1).

The difference between the extracted source parameters obtained by previously discussed methods does not exceed $4 \%$.

Figure 4 shows the measured source radii (left plot) and correlation strength parameters (right plot) of positive (red solid circles) and negative (blue empty circles) kaon pairs obtained for $600 \mathrm{GeV} / c \Sigma^{-}$, $\pi^{-}$and $540 \mathrm{GeV} / c$ p interactions with carbon and copper nuclei. The uncertainty due to the fit range was estimated by varying the upper limit from $q_{\text {inv }}=1 \mathrm{GeV} / c$ to $q_{\text {inv }}=0.6 \mathrm{GeV} / c$ and lower limit from $q_{i n v}=0 \mathrm{GeV} / c$ to $q_{i n v}=0.2 \mathrm{GeV} / c$, and did not exceed $6 \%$. 


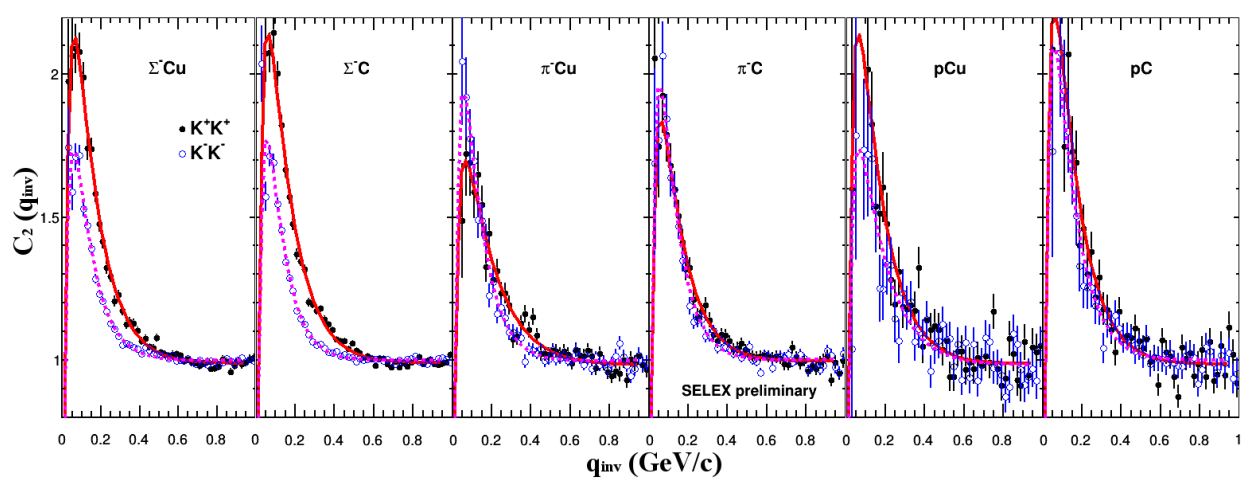

Figure 3. The positive (black solid circles) and negative (blue empty circles) kaon double ratios obtained for $\Sigma^{-} \mathrm{Cu}$ (a) and $\Sigma^{-} \mathrm{C}$ interactions. The solid red and dashed magenta lines represent fits performed using 1 of positively and negatively charged kaon pairs, respectively.
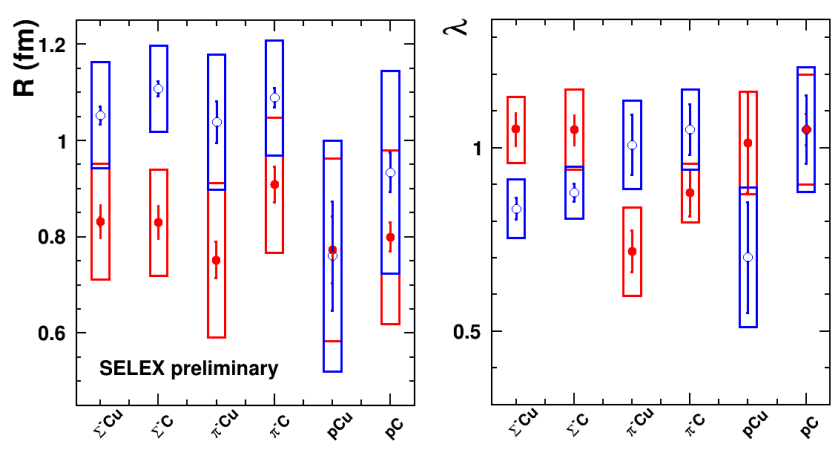

Figure 4. The dependence of the emitting source radii (left) and correlation strength parameter (right) on the type of the $\mathrm{h}+\mathrm{A}$ collision. The red solid and blue empty circles represent the estimated source parameters for positive and negative kaons, respectively. Boxes represent systematic uncertainties on the measured values.

The systematic errors (boxes) on $R$ and $\lambda$ for each beam type and target material are taken as the root mean square spread of the values calculated for the different sources of the systematic uncertainty.

Due to the limited acceptance and statistics it is not possible to select events with different multiplicity (collision centrality) classes for each collision type. In the current analysis, the extracted source radii for $600 \mathrm{GeV} / c \Sigma^{-}, \pi^{-}$and $540 \mathrm{GeV} / c$ p interactions with $\mathrm{Cu}$ and $\mathrm{C}$ nuclei are consistent within uncertainties and do not depend on the target material. The source radii of negatively charged kaons measured for $\Sigma^{-}$and $\pi^{-}$beams are generally larger than those for positive kaons. For $\Sigma^{-}$induced interactions the negative kaon correlation functions are smaller than for positive kaons at $q_{i n v} \leq 0.6 \mathrm{GeV} / c$. The $\lambda$ parameters obtained for positive and negative kaons for different hadron beams are similar for carbon and copper nuclei. 


\section{Summary}

We have presented the results from the SELEX experiment on positive and negative kaon femtoscopic measurements in $600 \mathrm{GeV} / c \Sigma^{-}, \pi^{-}$and $540 \mathrm{GeV} / c$ p interactions with $\mathrm{Cu}$ and $\mathrm{C}$ nuclei. The onedimensional source parameters were extracted for all $\mathrm{h}+\mathrm{A}$ collision types. The measured sizes of the emitting region for $\Sigma^{-} \mathrm{Cu}(\mathrm{C}), \pi^{-} \mathrm{Cu}(\mathrm{C})$ and $\mathrm{pCu}(\mathrm{C})$ interactions are similar and consistent with each other within uncertainties. The source radii of negatively charged kaons measured for $\Sigma^{-}$and $\pi^{-}$ beams are generally larger than those for positive kaons but consistent within the uncertainties. For $\Sigma^{-} \mathrm{Cu}(\mathrm{C})$ interactions the correlation functions of negative kaons are smaller than those for positive kaons at $q_{i n v} \leq 0.6 \mathrm{GeV} / c$.

\section{Acknowledgments}

Authors would like to thank the support from National Research Nuclear University MEPhI in the framework of the Russian Academic Excellence Project (contract No. 02.a03.21.0005, 27.08.2013).

\section{References}

[1] G. Goldhaber et al., Phys. Rev. 120, 300 (1960)

[2] J.S. Russ et al., Proc. Int. Conf. on High Energy Phys. II, 1259 (1998)

[3] U. Dersch et al., Phys. Lett. B 579, 277 (2000)

[4] J. Engelfried et al., Nucl. Instr. and Meth. A 409, 53 (1998)

[5] G.A. Nigmatkulov et al., Phys. Lett. B 753, 458 (2016)

[6] R. Lednický et al., Phys. Lett. B 373, 30 (1996)

[7] M.G. Bowler, Phys. Lett. B 270, 69 (1991)

[8] Yu.M. Sinyukov et al., Phys. Lett. B 432, 248 (1998)

[9] H. Bøggild et al., Phys. Lett. B 302, 510 (1993)

[10] J. Adams et al., Phys. Rev. C 71, 044096 (2005)

[11] T. Sjöstrand, S. Mrenna, P. Skands, JHEP 05, 026 (2006)

[12] P. Skands, Phys. Rev. D 82, 074018 (2010)

[13] K. Aamodt et al., Phys. Rev. D 82, 052001 (2010) 